\title{
On-chip 2D beam-steering with a liquid-crystal- tunable optical phased array
}

\section{Kazuki Nakamura}

Panasonic

Akira Hashiya

Panasonic Corporation

\section{Takaiki Nomura}

Panasonic

\section{Yoshikazu Yamaoka}

Panasonic

Masahiko Tsukuda

Panasonic

Hiroyuki Takagi

Panasonic

Yoshiki Sasaki

Panasonic

Atsushi Ishikawa

Panasonic

\section{Daivid Fowler}

University Grenoble Alpes and CEA

\section{Stephane Malhouitre}

University Grenoble Alpes and CEA

\section{Viviane Muffato}

University Grenoble Alpes and CEA

\section{Wilfried Rabaud}

University Grenoble Alpes and CEA

\section{Kazuya Hisada}

Panasonic

\section{Taku Hirasawa}

Panasonic (Japan)

Yasuhisa Inada ( $\sim$ inada.yasuhisa@jp.panasonic.com )

Panasonic (Japan) 
Keywords: Dynamic Beam-steering, Bragg-reflector Waveguide Outcoupler, Silicon Photonics

Posted Date: October 1st, 2020

DOI: https://doi.org/10.21203/rs.3.rs-78673/v1

License: (c) (1) This work is licensed under a Creative Commons Attribution 4.0 International License. Read Full License 


\section{Abstract}

Optical phased arrays (OPAs) with the ability of dynamic beam-steering hold great promise for industrial applications. Although they potentially offer high-resolution, high-speed and wide-angle beam-steering, 2D beam-steering has only been achieved by 1D OPAs with wavelength-tuning or by 2D OPAs containing enormous numbers of elements, thereby significantly increasing the system complexity and power consumption. Here we demonstrate a liquid-crystal-tunable Bragg-reflector waveguide outcoupler integrated with a 1D OPA. The output beam angle is controlled by an electric-field applied to the liquid crystal between the Bragg-mirrors, independently from steering along a second axis with the OPA. Our proof-of-concept demonstration with an 8-channel OPA shows 2D beam-steering with a $16^{\circ} \times 15^{\circ}$ field-ofview and $12 \mathrm{~ms} \times 14 \mu$ s time-response. Our approach opens a practical way for scalable OPAs capable of single wavelength 2D beam-steering, as well as demonstrating a strategy for the integration of liquid crystal materials with silicon photonics.

\section{Main Text}

Integrated optical phased arrays (OPAs) [1-13], inspired by phased-array radars, perform versatile beamsteering, such as random-access pointing and focusing on regions of interest, as well as sequential scanning. They are suitable for applications such as LiDAR [3-13], image projection [3,14-16] and optical communication $[12,17,18]$. In contrast to mechanical scanning, OPAs have the additional advantage of being compact and capable of high-speed response, allowing their functionality to be adapted to other systems such as 2D image sensors. A fusion of an agile beam-steering with an image sensor could realize 3D target tracking where the OPA beam focuses on objects that are identified from the image. Such an adaptive control of a LiDAR beam could be also utilized for obtaining dense 3D point clouds with deep learning-based depth completion guided by an image [19]. The key performance requirement for such a beam-steering system is an output beam with low-divergence and widely steerable in twodimensions.

Integrated OPAs based on silicon photonics technology are being actively developed. While 1D-OPAs allow wide-angle beam steering in one-dimension, scanning in the second dimension has been usually achieved with widely tunable lasers $[5-9,13]$. Single wavelength beam-steering in two-dimensions is naturally achieved by 2D-OPAs with a variable phase in each pixel $[2,3,12]$. However, such an approach requires the enormous numbers of phase shifters, thereby significantly increasing the system complexity and power consumption. 2D OPAs also generate multiple grating-lobes due to the limited antenna density, resulting in a significant reduction of the main-beam power. Although advanced phase shifters such as electro-optic [18], liquid crystal [20], multi-pass thermo-optic [13] and microelectro-mechanical-system (MEMS)-actuated shifters [21] have been demonstrated, large-scale and dense integration in a 2D array still remains elusive. Liquid crystal-on-silicon (LCOS) spatial light modulators [22-24] are another approach for 2D beam-steering, but their slow response has severely hindered their applications. 
Here we propose a LC-tunable Bragg-reflector waveguide outcoupler integrated with a 1D OPA for 2D beam-steering. The principles of the beam-steering mechanism are illustrated in Fig. 1. The structure of the outcoupler is similar to a VCSEL $[25,26]$, but comprises LC as a tunable core sandwiched between two distributed Bragg reflectors (DBR) to support the guided modes (Fig. 1b). This mode is coupled out through the top DBR with a lower reflectivity compared to the bottom one. By applying an external electric field to the LC core, the effective index of the guided mode is modulated, thereby dynamically changing the angle of the output beam from the outcoupler (Fig. 1c,d). The steering in the second dimension is obtained by building an array of outcouplers with controllable phases (Fig. 1b). This can be realized by connecting waveguide arrays with phase shifters (1D OPA) to the LC-tunable outcouplers (Fig. 1a).

Different from conventional techniques, our approach enables independent control of the LC and OPA for beam-steering in two directions. The OPA steering is generally faster $(<100 \mathrm{~ms}$ using thermal phase shifters) than the LC-tuning ( $10 \mathrm{~ms}$ ), resulting in beam-steering over a 2D field-of-view (FOV) within tens of milliseconds. Such a time response is sufficient for various applications, including imaging-based fusion systems and image projections where the frame rate is typically $\sim 30$ frames per second.

More importantly, the LC-tunable 1D OPA consists of a small number of elements compared to a conventional 2D OPA, thus greatly simplifying the driving system and reducing power consumption. The 1D array generates only a few OPA grating-lobes, which could even be completely suppressed by reducing the antenna pitch [4,27], leading to a large power fraction in the main beam. The large antenna pitch used in 2D OPAs means that they suffer severely from a reduction in the main beam power caused by the presence of multiple grating-lobes [2,3,12], which poses challenges for long-range applications.

Figure 2 shows the on-chip integration design of the LC-tunable 1D OPA based on silicon nitride waveguides operating at I $=940 \mathrm{~nm}$ (see Methods). The photonic circuit fabricated on the bottom DBR is coupled to the LC-tunable outcouplers, which are formed by a LC-filled core between the top and bottom DBRs. The outcoupler supports a high order DBR-guided mode in the vertical direction, while the lateral confinement is obtained by $\mathrm{SiO}_{2}$ blocks acting as low-index claddings (Fig. 2c). This mode has a different propagation constant and field profile with respect to the input waveguide mode (Fig. $2 \mathrm{~b}$ ). To manage the optical transfer between these waveguides, a grating-based mode converter $(\mathrm{MC})$ is introduced at the end of the input waveguide (Fig. 2a). Resonant mode conversion can be obtained via the phase matching condition $p=\mathrm{l} /\left(n_{\mathrm{w}}-n_{\mathrm{g}}\right)$ [28], where $p$ denotes the grating period, I the wavelength, $n_{\mathrm{w}}$ and $n_{\mathrm{g}}$ the effective index of the input and guided mode within the $\mathrm{MC}$ region. The exact resonance can be found by electrically tuning the LC index to 1.6 in the MC (Fig. 2d). Further optimization of the mode conversion efficiency is achieved by adjusting the grating diffraction strength and the field overlap between these modes [28].

To demonstrate LC-tunable beam-steering, we firstly fabricated and characterized a single Bragg-reflector waveguide outcoupler (Fig. 3). Fig. 3a shows the sample, which was fabricated using a modified CMOScompatible silicon photonics process, followed by integration of the LC (Supplementary Fig. S1). The LCtunable outcoupler was formed by introducing the LC into the gap between the top and bottom DBRs 
(Supplementary Table S1 for the DBR design and Fig. S4 for the calculated reflectivity). The LC orientation was then altered by applying an external field (Methods) as shown in polarized microscope images (Fig. 3b,c). The near-field (far-field) emission images are shown in Fig. 3d,e (Fig. 3f,g), demonstrating that the measured FWHM beam divergences in the far-field are close to the diffraction limited values. Fig. $3 \mathrm{~h}$ shows the beam-steering performance as a function of the applied voltage. The measured steering range was $\sim 30^{\circ}$ with a beam divergence of $\sim 0.25^{\circ}$. The small variation in beam divergence was attributed to the scattering loss depending on the propagation constant of the guided mode in the LC core. From the measured steering range, our simulations indicate that the index-tuning range was 1.65-1.54, although the ideal tuning range provided by the birefringence is $1.68-1.52$. This small discrepancy is because the movement of the LC inside the small core is limited (Supplementary Fig. S8).

We then demonstrated a LC-tunable OPA for 2D beam-steering (Fig. 4). The sample was prepared by adding LC material onto a Si-chip containing 1D OPA, consisting of a waveguide array and thermal phase shifters. Figure 4a shows a microscope image of the sample with an 8-channel array of LC-tunable outcouplers, and Fig. 4b shows an image of the near-field emission. As shown in Fig. 4c, a beam spot in the far-field was clearly visible after calibrating the initial phases (Supplementary Fig. S6). Figure 4c demonstrates 2D beam-steering with a FOV of $16^{\circ} \times 15^{\circ}$ achieved by tuning the phase shifters in the $y$ direction and the $L C$ in the $x$-direction. The grating-lobes at $\pm 15^{\circ}$ due to the relatively large outcoupler pitch $(3.6 \mathrm{~mm})$ were generated only in the y-direction. The weak scattered light seen in the $x$-direction is likely due to the defects on the bottom of trench for the LC core (Supplementary Fig. S5e). The FOV of $16^{\circ}$ obtained by the LC-tuning was smaller than that of the single outcoupler $\left(30^{\circ}\right)$. This was caused by the limited movement of the LC inside the small core width (Supplementary Fig. S8). Solutions to these problems include the use of a LC slab-waveguide design (Supplementary Fig. S9), a smaller OPA pitch to suppress the grating-lobes, and a larger LC area enabling improved LC control. The response time of the LC tuning was $12 \mathrm{~ms}$, as shown in Supplementary Fig. S7b. The LC-tuning required extremely low power $(<100 \mathrm{~mW})$ since the liquid-crystal itself is essentially a capacitive load. The thermal phase shifters showed a response time of $14 \mathrm{~ms}$ and required $80 \mathrm{~mW}$ each for $\mathrm{p}$ phase shift (Supplementary Fig. S7a). In the future, more advanced phase shifters $[13,18]$ may be used to reduce the power consumption, realizing low-power operation even for a larger scale LC-tunable OPA.

In conclusion, we have proposed a LC-tunable 1D OPA on a Si-chip for continuous 2D beam-steering at a fixed wavelength. We have demonstrated LC-tunable beam steering using an integrated single LC-tunable Bragg-reflector waveguide outcoupler. By connecting the array of LC-tunable outcouplers to an 1D OPA, beam-steering in two-dimensions was additionally obtained. Our device with an 8-channel OPA demonstrates 2D beam-steering with $16^{\circ} \times 15^{\circ} \mathrm{FOV}$ and $12 \mathrm{~ms} \times 14 \mu \mathrm{s}$ response time at I $=940 \mathrm{~nm}$. We believe the proposed LC-tunable OPAs hold great promise for industrial applications. Our LC integration method is applicable to conventional photonic integrated circuits and provides a new platform allowing the combination of the advantages of silicon photonics and the large refractive index modulation and low power operation of LCs. 


\section{Methods}

Simulations. The finite-element method (Synopsys, FemSIM) was used to simulate the effective refractive indices and the mode profiles of the waveguides and the LC/Bragg-reflector waveguides, while the eigenmode expansion propagation method (Synopsys, ModePROP) was used to simulate the mode conversion.

Device design. The mode converter is designed for a wavelength of $940 \mathrm{~nm}$ with a refractive index of 2 for $\mathrm{SiN}$ waveguide core and 1.46 for the $\mathrm{SiO}_{2}$ cladding. To reduce propagation loss, the input waveguides need to be separated from both DBRs, which contain high index layers. Therefore, we adopted an outcoupler design that has a large separation between the top and bottom DBRs. The width of the waveguide is $600 \mathrm{~nm}$ but is gradually expanded near the mode conversion to match the width of the LCcore. The core width is $10 \mathrm{~mm}$ for the single Bragg-reflector waveguide outcoupler and $1.6 \mathrm{~mm}$ for the LCtunable OPA with the pitch of $3.6 \mathrm{~mm}$. For the mode conversion, a grating with the period $690 \mathrm{~nm}$ and the depth of $90 \mathrm{~nm}$ is formed, followed by encapsulation with $\mathrm{SiO}_{2}$. The other design parameters are as follows: waveguide height $=300 \mathrm{~nm}, \mathrm{SiO}_{2}$ underclad thickness $=900 \mathrm{~nm}, \mathrm{LC}$-core height $=2470 \mathrm{~nm}, \mathrm{SiO}_{2}$ cladding height $2000 \mathrm{~nm}$, reflectivity of top DBR $=99.6 \%$, reflectivity of bottom DBR $=99.96 \%$.

LC and OPA driving. To tune the LC, a square wave voltage with a frequency of $3 \mathrm{kHz}$ was applied to the electrodes. The LC orientation was controlled as a function of the square-wave amplitude. The phaseshifter in the OPA was composed of a TiN heater near the SiN waveguide. A DC voltage was applied using constant voltage circuits to operate the phase-shifters.

\section{Declarations}

\section{Acknowledgement}

We thank F. Koyama and X. Gu for help with Bragg-reflector waveguide simulations and discussions. A part of this work was supported by Kyoto University Nano Technology Hub in "Nanotechnology Platform Project" sponsored by the Ministry of Education, Culture, Sports, Science and Technology (MEXT), Japan.

\section{Author contributions}

Y.I. conceived and designed the project. K.N. and A.H. designed and characterized the sample. Y.Y., M.T, and Y.S. developed the basic fabrication process for the bottom substrate, and T.N. and H.T. developed 
the liquid crystal integration process. D.F. designed the 1D OPA, and S.M. and W.R. developed fabrication process of the OPA on DBR. K.H. and T.H. supervised the whole project. K.N. and Y.I. wrote the most part of paper with the help of other authors. All the authors discussed the results and contributed to the manuscript.

\section{Competing interests}

The authors declare no competing interests.

\section{References}

1. Von Acoleyen, K. et al. Off-chip beam steering with a one-dimensional optical phased array on silicon-on-insulator. Opt. Lett. 34, 1477-1479 (2009).

2. Von Acoleyen, K., Rogier, H. \& Baets, R. Two-dimensional optical phased array antenna on silicon-oninsulator. Opt. Express 18, 13655-13660 (2010)

3. Sun, J., Timurdogan, E., Yaacobi, A. et al. Large-scale nanophotonic phased array. Nature 493, 195199 (2013).

4. Yaacobi, A. et al. Integrated phased array for wide-angle beam steering. Opt. Lett. 39, 4575-4578 (2014)

5. Kim, T. et al. A single-chip optical phased array in a wafer-scale silicon photonics CMOS 3Dintegration platform. IEEE J. Solid-state Circuits 54, 3061-3074 (2019)

6. Doylend, J. K. et al. Two-dimensional free-space beam steering with an optical phased array on silicon-on-insulator. Opt. Express 19, 21595-21604 (2011)

7. Hulme, J. C. et al. Fully integrated hybrid silicon two dimensional beam scanner. Opt. Express 23, 5861-5874 (2015)

8. Hutchison, D. N. et al. High-resolution aliasing-free optical beam steering. Optica 3, 887-890 (2016)

9. Kwong, D. et al. On-chip silicon optical phased array for two-dimensional beam steering. Opt. Lett. 39. 941-944 (2014)

10. Chung, S., Abediasl, H. \& Hashemi, H. A monolithically integrated large-scale optical phased array in silicon-on-insulator CMOS. IEEE J. Solid-State Circuits 53, 275-296 (2018)

11. Tyler, N. A. et al. SiN integrated optical phased arrays for two-dimensional beam steering at a single near-infrared wavelength. Opt. Express 27, 5851-5858 (2019)

12. Fatemi, R., Khachaturian, A. \& Hajimiri, A. A nonuniform sparse 2-D large-FOV optical phased array with a low-power PWM drive. IEEE J. Solid-State Circuits 54, 1200-1215 (2019) 
13. Miller, S. A. et al. Large-scale optical phased array using a low-power multi-pass silicon photonic platform. Optica 7, 3-6 (2020)

14. Aflatouni, F., Abiri, B., Rekhi, A. \& Hajimiri, A. Nanophotonic projection system. Opt. Express 23, 21012-21022 (2015)

15. Raval, M., Yaacobi, A. \& Watts, M. R. Integrated visible light phased array system for autostereoscopic image projection. Opt. Lett. 43, 3678-3681 (2018)

16. Shin, M. C. et al. Chip-scale blue light phased array. Opt. Lett. 45, 1934-1937 (2018)

17. Abediasl, H. \& Hashemi, H. Monolithic optical phased-array transceiver in a standard SOI CMOS process. Opt. Express 23, 6509-6519 (2015)

18. Poulton, C. V. et al. Long-range LiDAR and free-space data communication with high-performance optical phased arrays. IEEE J. Sel. Top. Quantum Electron 25, 1-8 (2019)

19. Bergman, A. W., Lindell, D. B. \& Wetzstein, G. Deep Adaptive LiDAR: End-to-end Optimization of Sampling and Depth Completion at Low Sampling Rates. Proc. IEEE ICCP, 1-11 (2020)

20. Notaros, J., Notaros, M., Raval, M. \& Watts, M. R. Liquid-crystal-based visible-light integrated optical phased arrays. Conference on Lasers and Electro-Optics (CLEO), 1-2 (2019)

21. Wang, Y. et al. 2D broadband beamsteering with large-scale MEMS optical phased array. Optica 6, 557-562 (2019).

22. Resler, D. P., Hobbs, D. S., Sharp, R. C., Friedman, L. J. \& Dorschner, T. A. High-efficiency liquid-crystal optical phased-array beam steering. Opt. Lett. 21, 689-691 (1996)

23. Beeckman, J., Neyts, K. \& Vanbrabant, P. Liquid-crystal photonic applications. Opt. Eng. 50, 081202081217 (2011).

24. Zhang, Z., You, Z. \& Chu, D. Fundamentals of phase-only liquid crystal on silicon (LCOS) devices. Light Sci App/3, e213 (2014)

25. Gu, X. et al. Beam steering in GalnAs/GaAs slow-light Bragg reflector waveguide amplifier. Appl. Phys. Lett. 99, 21107-1-3 (2011)

26. Gu, X., Shimada, T. and Koyama, F. Giant \& high-resolution beam steering using slow-light waveguide amplifier. Opt. Express 19. 22675-22683 (2011)

27. Phare, C. T., Shin, M. C., Miller, S. A., Stern, B. \& Lipson, M. Silicon optical phased array with highefficiency beam formation over 180 degree field of view. Preprint at https://arxiv.org/abs/1802.04624 (2018).

28. Jin, W. \& Chiang, K. S. Mode converters based on cascaded long-period waveguide gratings. Opt. Lett. 41. 3130-3133 (2916)

\section{Figures}



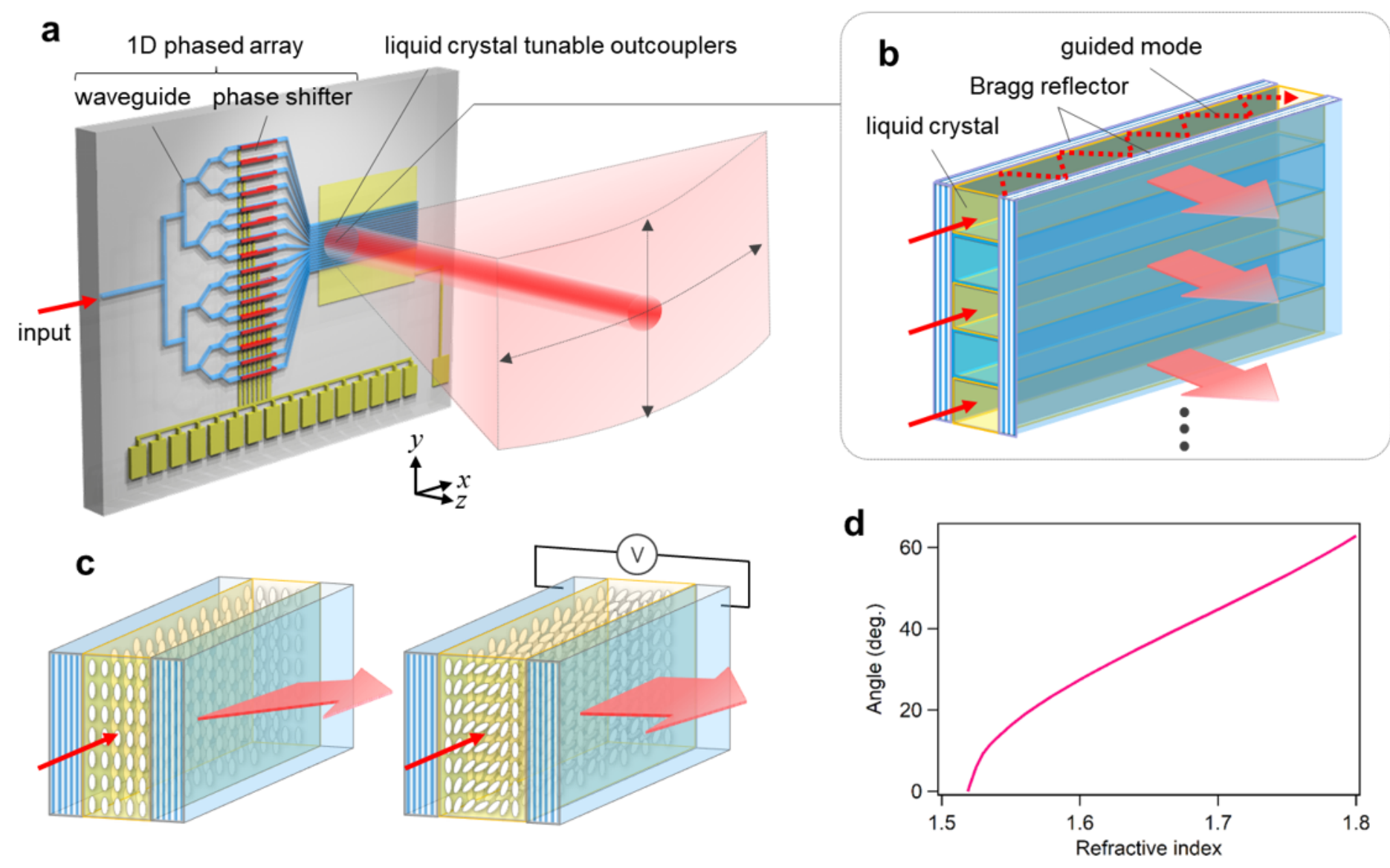

\section{Figure 1}

Liquid-crystal (LC) tunable Bragg reflector waveguide outcoupler array for 2D Beam-Steering. a, Schematic of an on-chip 2D beam-steering device. Input laser light is guided to the LC-tunable Bragg reflector outcouplers through the waveguides with phase-shifters. The output beam is then steered in the $x$-direction with the tunable LC and in the y-direction with the phase shifters. b, Schematic of the outcoupler array. The guided light propagates along the $x$-axis (illustrated as a dotted line) in the LC core between the top and bottom DBRs and is emitted though the top DBR. The emissions from the outcoupler array interfere in the far-field and form a beam that can be steered using the phase shifters. $\mathrm{C}$, Structure of the LC-tunable Bragg reflector outcoupler. The angle of the output beam in the xz-plane can be controlled by tuning the refractive index of the LC for the TE guided mode with an external electric field. $d$, Calculated angle of the output beams as a function of the refractive index of the LC. 


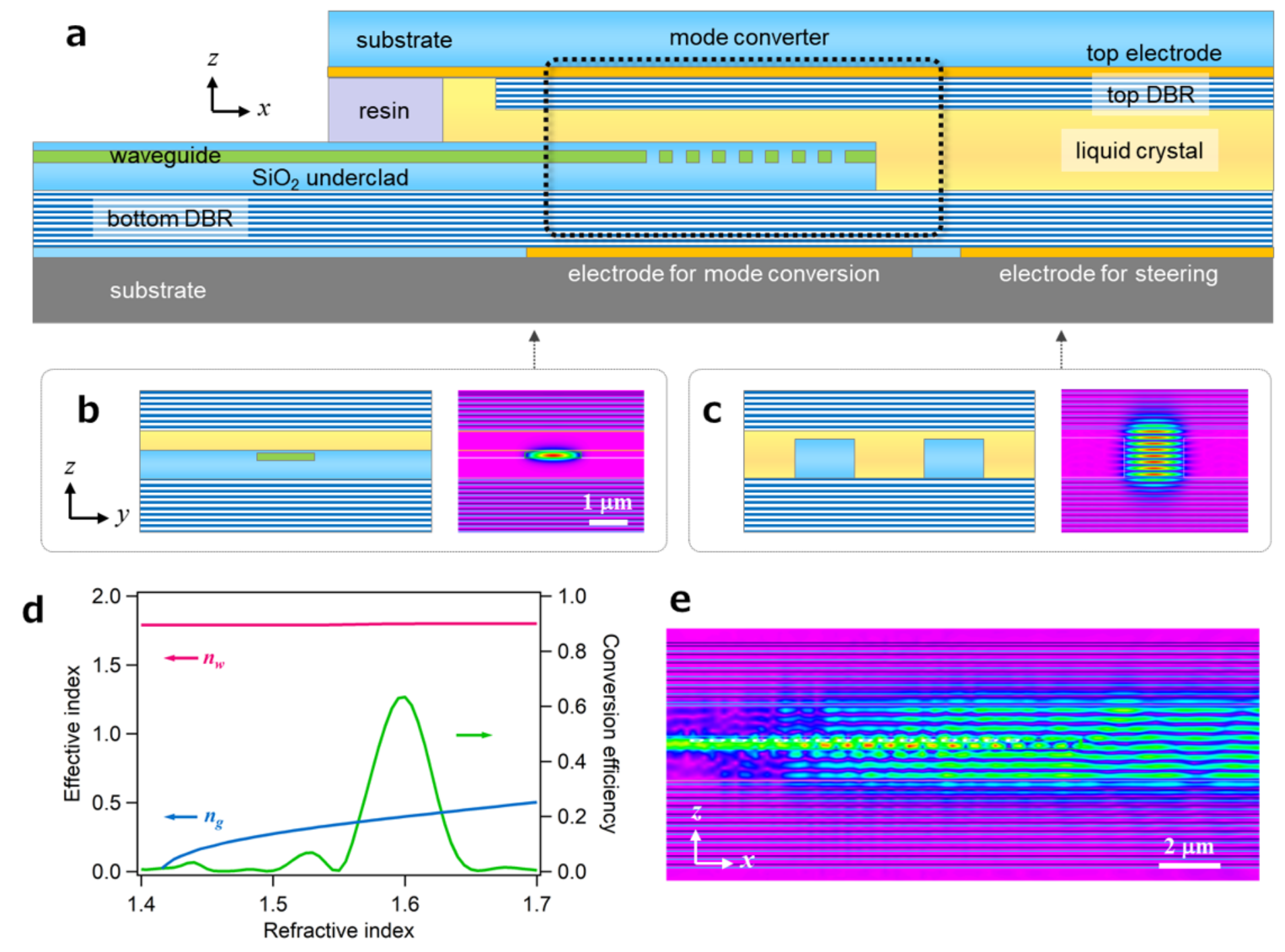

\section{Figure 2}

On-chip integration of LC-tunable Bragg reflector waveguide outcouplers with an OPA. a, Cross-sectional schematic of a connection between the waveguide and the LC-tunable outcoupler in the xz-plane. A grating-based mode converter is placed at the end of the waveguide. Top and bottom electrodes are introduced to apply an external electric field. The bottom electrode is patterned to drive the LC for mode conversion and beam-steering separately. $b$, The structure of the waveguide and $c$, the Bragg reflector waveguide in the yz-plane, and the corresponding simulated mode profiles. $d$, The calcu-lated effective index of the waveguide (nw) and the DBR-guided (ng) mode at the mode transfer region. The calculated mode conversion efficiency of the grating is also shown. Resonant mode conversion is achieved by tuning the LC index to $~ 1.6$ where the phase matching condition is satisfied. e, The intensity profile at the mode conversion area. The waveguide mode is efficiently transferred to the DBR-guided mode on the resonance. 

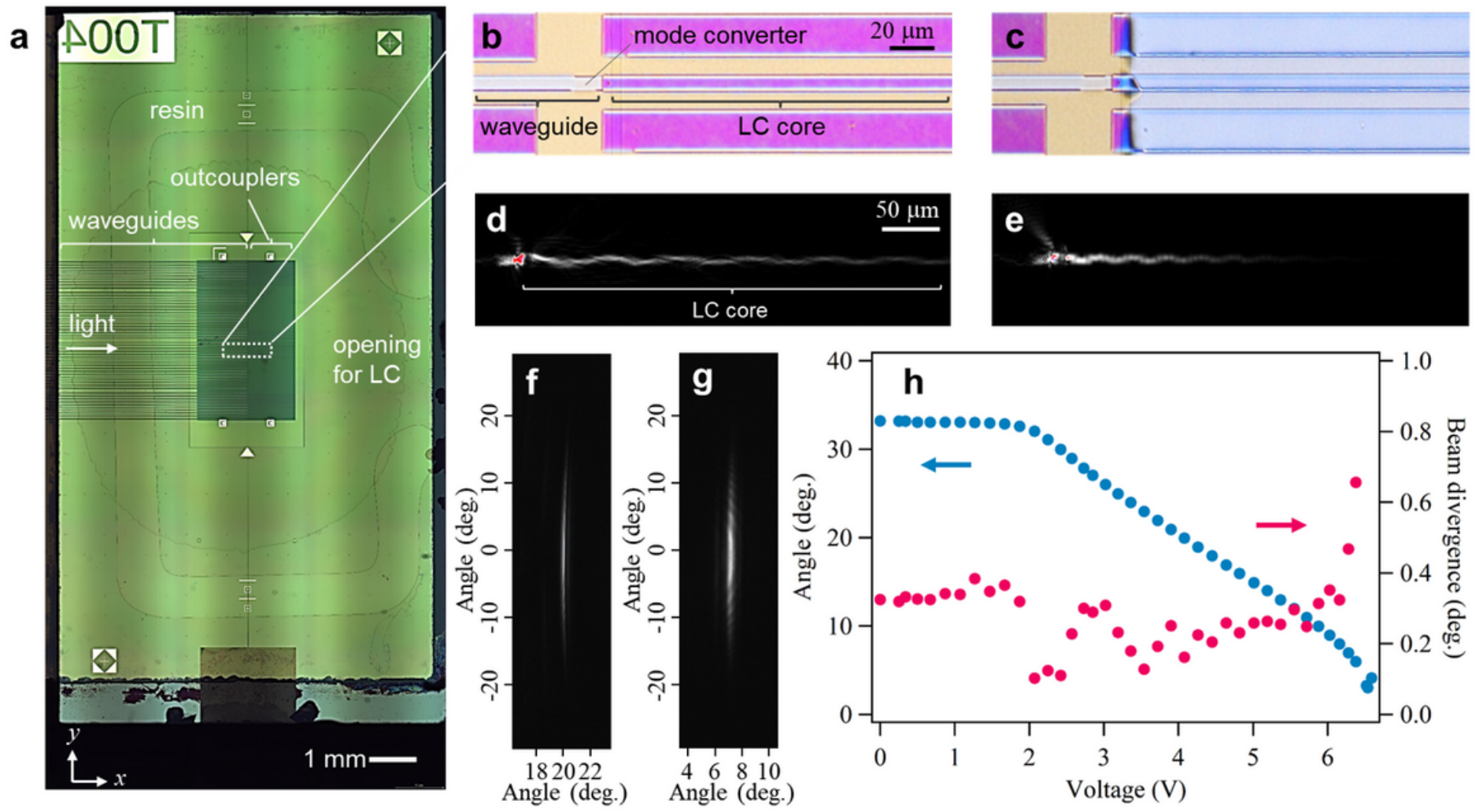

Figure 3

Demonstration of a single LC-tunable Bragg reflector waveguide outcoupler. a, Mi-croscope image of our sample with single outcouplers. After fabricating the top and bottom substrates, they were bonded with a UV curing resin, forming a C-shaped pattern. The LC was then injected from the opening of the pattern, which was finally sealed with the same resin. b, Polarized microscope image of a LC core with an applied voltage of $0 \mathrm{~V}$ and $\mathrm{c}, 6.3 \mathrm{~V}$. The color change from pink to blue corresponds to the change of the LC orientation. d, Near-field emission images at $4.1 \mathrm{~V}$ and e, 6.3 V. The propagation length (140 $\mathrm{mm}$ at $4.1 \mathrm{~V})$ became short (47 $\mathrm{mm}$ at $6.3 \mathrm{~V}$ ) where the emission angle is close to the normal. $\mathrm{f}$, Far-field images at $4.1 \mathrm{~V}$ and $\mathrm{g}, 6.3 \mathrm{~V}$. The measured FWHM of the beam divergence angles were $0.16^{\circ} \times 7.6^{\circ}$ at $4.1 \mathrm{~V}\left(0.47^{\circ} \times 7.1^{\circ}\right.$ at $6.3 \mathrm{~V})$, which are close to the diffraction limited values of $0.16^{\circ} \times 7.1^{\circ}\left(0.45^{\circ} \times 7.1^{\circ}\right)$ for a mode with the field size of $4.9 \square \mathrm{m}$ and a propagation length of $140 \mathrm{Jm}(47 \mathrm{\square m})$ in the LC core. $\mathrm{h}$, The emission angle and the beam divergence as a function of the applied voltage. The beam is steered by $30^{\circ}$ with a beam divergence of $\sim 0.25^{\circ}$. 

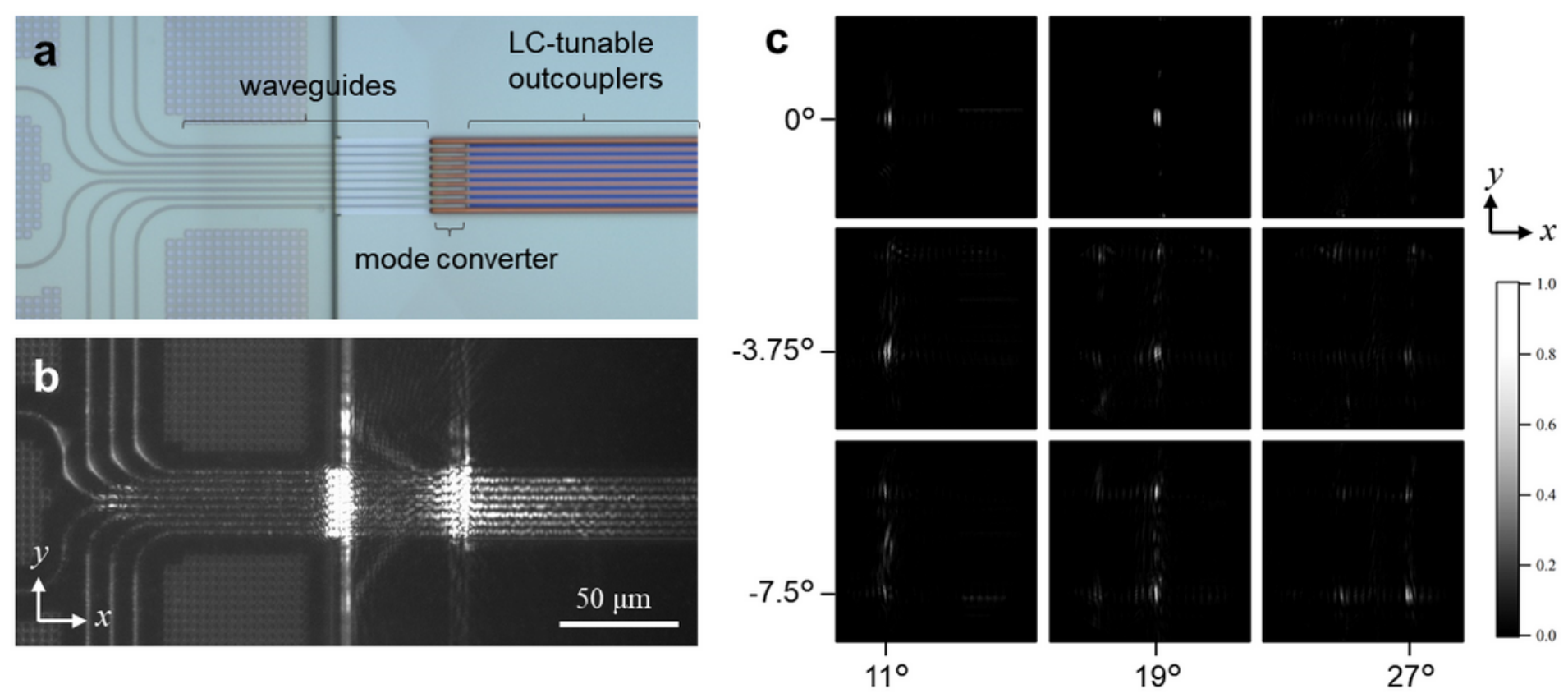

Figure 4

Demonstration of a LC-tunable optical phased array. a, Microscope image of the 8-channel array of waveguides, mode converters and LC-tunable outcouplers. The LC cores (dark blue) were formed between the SiO2 claddings (brown). b, Near-field image of the emission from the outcoupler array with the same view as a. The outcoupler array emission from the LC cores are clearly visible in the image. The unwanted scattered light is also seen from the mode converter and the edge of a SiO2 cover layer. c, Far-field emission patterns from the same outcoupler array. The measured beam divergence was $0.38^{\circ} \times 2.6^{\circ}$, which was larger than the diffraction limit $\left(0.37^{\circ} \times 1.9^{\circ}\right)$ in the $y$-direction due to the intensity variation in the output power from each outcoupler. By tuning the phase shifters (LC), the output beam is steered in the $x$-direction (y-direction) over a range of $15^{\circ}\left(16^{\circ}\right)$.

\section{Supplementary Files}

This is a list of supplementary files associated with this preprint. Click to download.

- supplementary2Dsteering0915.docx 